ossicular defect (as expected) and an OCC extending from the stapedial tendon to the promontory. We removed the entire OCC and reconstructed the ossicular chain with tragal cartilage. The postoperative air-bone gap was $9.4 \mathrm{~dB}$.

Conclusions: OCC often presents in a way very similar to $\mathrm{COA}$, with conductive hearing loss and an intact tympanic membrane. However, a history of progressive hearing loss and the presence of a subtle soft tissue density on TBCT are suspicious of OCC, rather than COA, in which the hearing loss is of a congenital nature. In this patient, the cholesteatoma was located in the sinus tympani around the stapedial tendon and was difficult to assess with an operating microscope. In lesions of the sinus tympani and facial recess, endoscope-assisted microsurgery can facilitate cholesteatoma removal and reduce the risk of recurrence.

doi:10.1017/S0022215116005715

\section{ID: IP075}

Interaction between keratinocytes and fibroblasts induces osteoclastogenesis: a mechanism underlying cholesteatomainduced bone destruction

\section{Presenting Author: Yoriko Iwamoto}

Yoriko Iwamoto, Yumi Ohta, Ryusuke Imai, Tetsuo Morihana, Hidenori Inohara

Osaka University

\section{Learning Objectives:}

Bone is a highly dynamic organ, which is maintained by a balance between bone-resorbing osteoclasts and boneforming osteoblasts. Increased osteoclast activity shifts the balance toward bone resorption, cause bone destructive diseases such as rheumatoid arthritis and periodontitis. Ectopic induction of receptor activator of nuclear factor kappa-B ligand (RANKL), a regulator of osteoclast differentiation, leads abnormal osteoclastogenesis. For example, in rheumatoid arthritis, synoviocyte is known as a major source of RANKL.

Cholesteatoma is a non-neoplastic lesion arising in middle ear, which consists of hyper keratinizing epithelial layer and fibrous connective tissue. Due to its bone destructive character, it can cause severe complications. However the mechanism of the bone destruction by cholesteatoma remains to be elucidated.

In this study, we established cholesteatoma-like mass composed of mouse ear pinna-derived keratinocytes and fibroblasts on the calvarial bone of mouse. Histological analysis revealed the experimental mass lesion induced osteoclastogenesis on the bone surface. In addition, we succeeded in establishing an in vitro coculture system of keratinocytes, fibroblasts and osteoclast precursors, and found that keratinocytes stimulate the induction of RANKL in fibroblasts, which leads to osteoclastogenesis.

Thus,this study demonstrates that interatction between keratinocytes and fibroblasts is involved in the differentiation of osteoclasts, which may provide the molecular vasis of a new therapeutic strategy for cholesteatomainduced bone destruction.
doi:10.1017/S0022215116005727

\section{ID: IP076}

LION Surgery Broadcasts: Communal viewing benefits with perceived quality of transmission, surgery and discussion

\section{Presenting Author: Steve Backhouse}

Vibha Jaiswal $^{1}$, Vibha Jaiswal ${ }^{1}$, Alice Davies ${ }^{2}$, Ryan Murphy ${ }^{2}$, Razun Miah ${ }^{2}$, David Owens ${ }^{3}$, Steve Backhouse ${ }^{4}$

${ }^{1}$ ENT Surgery, Wales Deanery, UK, ${ }^{2}$ Audiology

Department, Bridgend Hospital, Wales, UK,

${ }^{3}$ ENT Department, University Hospital of

Wales, Cardiff, UK, ${ }^{4}$ Chole

Learning Objectives: To improve otological surgical knowledge \& techniques.

The Live International Otolaryngology Network (LION) aims to promote high quality medical and continuous surgical education programmes, seeking to improve knowledge and skills of practicing otolaryngologists. LION's purpose is to achieve a worldwide permanent interactive network within ENT, promoting distant learning using videoconferencing technology. Theoretically internet webcasts provide a cost effective, environmentally friendly way for otolaryngologists to access CPD.

Preparations were made on the $12^{\text {th }}$ May 2015 In order to maximise this opportunity in South Wales for ENT surgeons and allied health professionals. We organised a 'communal' viewing to promote open discussion between allied professionals in attendance and assess the educational experience by targeted questionnaires to give validity for CPD accreditation by RCSEdinburgh and ENTUK. 15 delegates attended (5 ENT consultants, 6 ENT trainees, 1 ENT SAS, 2 audiologists \& 1 audiology student). $71 \%$ had watched a LION broadcast previously and all felt that the communal broadcast was better than viewing alone, that they would re-attend a communal LION broadcast in the future, and would recommend such to a colleague. Perceived broadcast transmission quality, surgical technique and discussion were generally good across both channels.

Communal viewing of the LION broadcast was well received by all delegates. The following improvements were suggested: wider advertisement, simultaneous screening of broadcast channel 1 and 2, and a second screen in each room for background information/ case studies. Although the numbers are small, feedback for individual surgeons and procedures will allow quality assurance and improvement for the next broadcast.

doi:10.1017/S0022215116005739

\section{ID: IP077}

Climbing up the learning curve in totally endoscopic cholesteatoma surgery

Presenting Author: Adrian James

Adrian James, William Parkes

University of Toronto 
Learning Objectives:

Introduction: Totally endoscopic ear surgery (TEES) facilitates cholesteatoma treatment giving enhanced visulation of middle ear recesses without the post-operative morbidity of open surgery. TEES is not as yet an acceptable option for all cases, or indeed all surgeons. This study reports on factors influencing the adoption of TEES in paediatric cholesteatoma by a single surgeon.

Methods: A paediatric cholesteatoma surgery database, maintained over an 11 year period, was searched to determine the proportion of cases completed by TEES or an open post-auricular approach each year. Comparison was made with factors potentially linked to the surgeon's ability to perform TEES including extent of cholesteatoma, patient age, availablity of equipment and attendance at endoscopic courses.

Results: Of 649 surgeries 86 were by TEES and 37 by endoscope transcanal with microscope-guided assistance. The proportion of TEES cases per year increased from 1/75 $(1 \%)$ to $35 / 57(61 \%)$ over the last 7 years. Empirically, this increase correlated with acquistion of specialised instruments and attendance at courses. Overall, TEES cases were less extensive (median Mills stage: 1 versus $2 ; \mathrm{p}<0.001$, MannWhitney) but similar in age (median $11.5 \mathrm{v} 10.8 \mathrm{yr}$ NS, MannWhitney). Over the last 50 open cases, extent of disease $(54 \%)$, narrow ear canal $(30 \%)$ and defect too large for reconstruction $(12 \%)$ were noted as the commonest factors for utilising an open approach. Most recently, endoscopic canalplasty and non-autogenous grafts have further increased the range of TEES.

Conclusions: Cholesteatoma can be treated in a majority of children with TEES, but an open approach is still required when the ear canal provides inadequate access to the limits of the disease. The surgeon's ability to complete TEES is enhanced by appropriate training, acquistion of specialist instruments, motivation and experience. Development of surgical techniques is allowing a greater proportion of cases to be completed endoscopically.

\section{doi:10.1017/S0022215116005740}

\section{ID: IP078}

Extratympanic imaging of middle and inner ear structures of the mouse and rat model using optical coherence tomography(OCT)

\section{Presenting Author: Hee Jeong Jeong}

Hee Jeong Jeong, Se-Joon Oh, Soo-Keun Kong

Pusan National University Hospital

\section{Learning Objectives:}

Background and Objective: Noninvasive middle and inner ear imaging using Optical Coherence-Tomography (OCT) presents some unique challenges for real-time, clinical use in animals and humans. OCT has been used in other fields for obtaining high-resolution cross-sectional images of the tissue. The goal of this study was to investigate whether
OCT provides information about the middle and inner ear microstructures in both rats and mice by extratympanic approach.

Materials and Methods: Six BALB/c mice and Sprague Dawley rats were enrolled to the experiment, and to acquire an image of the entire tympanic membrane, the auricle and cartilaginous external auditory canal were removed, the swept-source OCT system was tested to identify the middle and inner ear microstructures. After that, the TM and bulla were removed to confirm whether more detailed middle and inner ear images might be obtained.

Results: It was possible to image through the tympanic membrane extratympanically and into the middle ear cavity involving several middle ear structures in both rats and mice. We could also obtain the inner ear images through the otic capsule and into the cochlea in the mice by extratympanic approach. However, the bulla should be removed to provide the inner ear structural images in the rats. The whole cochlea of the apical, middle and basal turn could be visualized and the bony thickness of the otic capsule covering the cochlea could also be measured simultaneously.

Conclusions: OCT is a promising technology to noninvasively assess middle ear and inner ear microanatomy in both mice and rats. These findings are meaningful because there were no previous report to describe the middle and inner ear structure looking by extratympanically. This imaging informations can be useful in the diagnosis of diseases of the middle and inner ear if it is clinically applied through further studies.

\section{doi:10.1017/S0022215116005752}

\section{ID: IP079}

\section{The Perioperative Strain Changes of Chronic Otitis Media Surgery}

Presenting Author: Su Hee Jeong

Su Hee Jeong, Soonil Yoo, Sungwoo Han, Geun Jeon Kim, Jin Bu Ha, Dong-Kee Kim

College of Medicine, The Catholic University, Korea

Learning Objectives: chronic otitis media culture strain.

Introduction: The perioperative prophylactic antibiotic of chronic otitis media (COM) surgery is selected based on the result of preoperative bacterial culture. Learning objectives: To investigate the changes of strains of middle ear through COM surgery, we conducted bacterial culture before, during, and after COM surgery. Method: We analyzed the medical records of 156 patients who received COM surgery at Daejeon St. Mary's Hospital from March 2012 to September 2015. Preoperative bacterial culture was conducted with otorrhea or swap of middle ear mucosa, intraoperative culture was conducted with granulation tissue in either the middle ear or mastoid cavity, and postoperative culture was selectively conducted when otorrhea was developed after surgery. Results: Sixty cases of tympanoplasty only and 96 cases of both tympanoplasty and mastoidectomy were performed for 111 cases of COM-without-cholesteatoma and 45 cases of COM-with-cholesteatoma. The growths of 